\title{
Das glymphatische System
}

\section{Susanne F. Dick-Wallace}

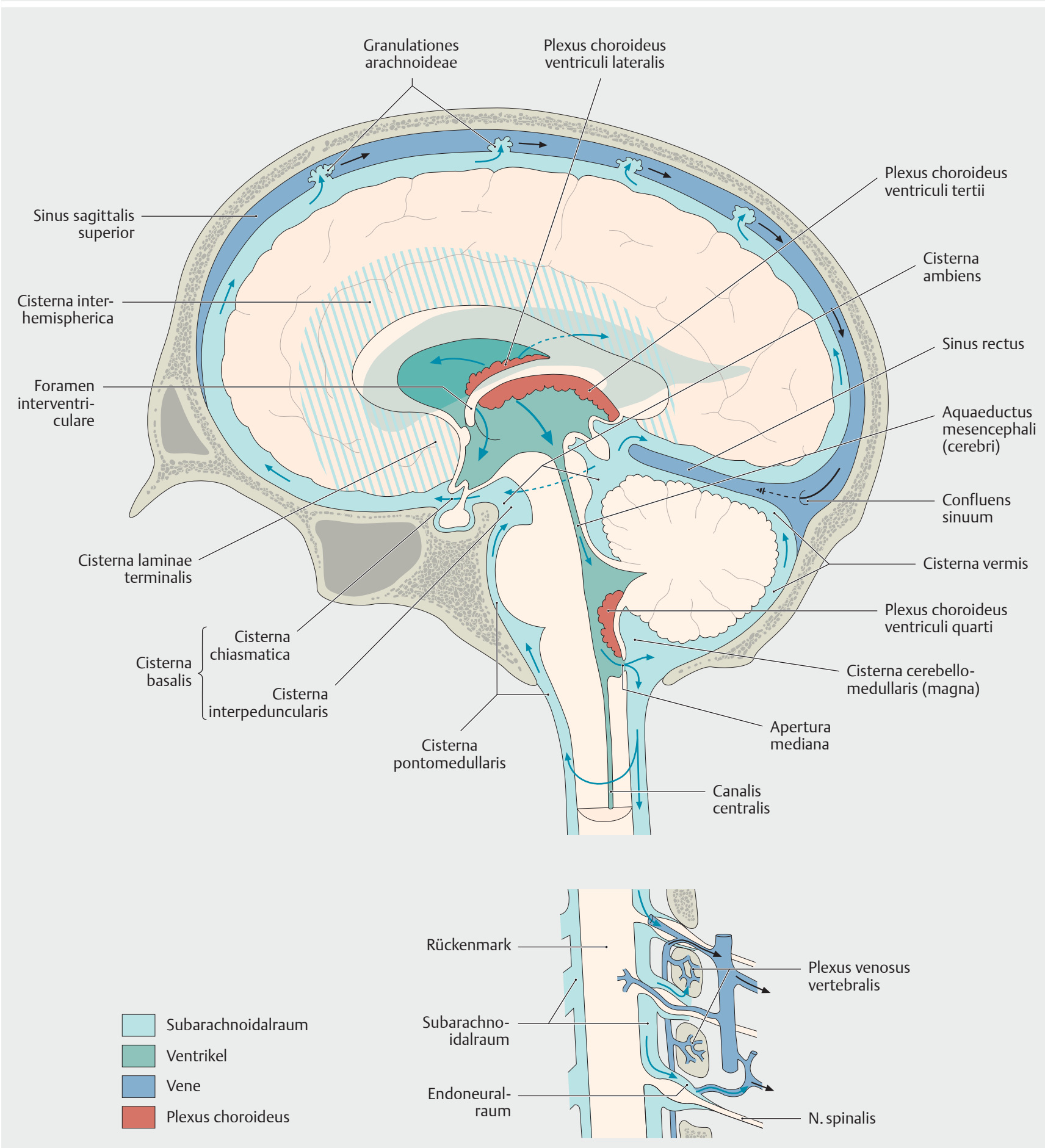

- Abb. 1 Liquorzirkulation. (Quelle: Schünke M, Schulte E, Schumacher U. Prometheus. LernAtlas der Anatomie. Kopf, Hals und Neuroanatomie. Illustrationen von M. Voll und K. Wesker. 5. Aufl. Stuttgart: Thieme; 2018) 
Im Jahr 2013 entdeckten Wissenschaftler ein effektives Drainagesystem im Gehirn. Im gesamten Gehirn werden die Blutgefäße von feinen Kanälen begleitet. Dieses Kanalsystem transportiert die aus dem Interstitium filtrierten Abfallstoffe ab. Die Innenwände der Kanäle bestehen v. a. aus Endothelzellen und glatten Muskelzellen. Die Außenwände werden von den flächigen Ausstülpungen (Endfüßchen) der Astrozyten gebildet, die Wasserkanäle enthalten Aquaporin 4. Die Astrozyten machen die größte Gruppe der Gliazellen aus. Durch die Ähnlichkeit zum Lymphsystem des Körpers tauften die Forscher das neu entdeckte System „glymphatisches System“ - zusammengesetzt aus „Glia“ und „Lymphe“.

\section{Das ZNS und die Lymphe}

Bis vor relativ kurzer Zeit wurde davon ausgegangen, dass das ZNS kein eigenes Lymphsystem hat und das periphere Lymphsystem mit dem Waldeyer-Rachenring bzw. den Tonsillen, Polypen und Lymphknoten im zervikalen Bereich an der Schädelbasis endet. Ab der Dura mater gäbe es kein Lymphsystem. Die Blut-Hirn-Schranke ( $\bullet$ Abb. 2) und die Blut-Liquor-Schranke wurden unter physiologischen Bedingungen als unüberwindliche Grenze betrachtet, nur wenige Stoffe seien durchgängig. Die Hypothese war, dass die „Abfallbeseitigung“ auf individuellem Zellniveau stattfindet und der Liquor cerebrospinalis dem lymphatischen System entspricht. Es stellt sich die Frage, wie das metabol hochgradig aktive ZNS seine Abfallund Giftstoffe entsorgt.

2013 entdeckte die Forschergruppe um Nedergaard [2] einen physiologischen Mechanismus im Bereich der Cliazellen des ZNS, den sie in Anlehnung an das lymphatische System glymphatisches System tauften. Sie waren die Ersten, die diese funktionelle Einheit beschrieben. Ähnlich dem lymphatischen System wird das glymphatische System als fließendes Durchlaufsystem zum Abtransport von überflüssigem und schädlichem Material verstanden. Die Transportflüssigkeit wird in das lymphatische System abgegeben.

Louveau und Kipnis [3] sowie Aspelund et al. [10] entdeckten später auf der Suche nach Zugangswegen für T-Zellen die Arteriolen und Venolen begleitende Lymphgefäße. Sie fanden, dass die duralen Sinus und die Aa. meningeae mediae von konventionellen Lymphgefäßen begleitet werden, die eine Verbindung zum glymphatischen System herstellen. Sie verbinden das glymphatische System mit dem meningealen Gebiet und drainieren Immunzellen, kleinere Moleküle und überschüssige Fluida, weshalb sie auch eine Rolle bei neuroinflammatorischen bzw. neurodegenerativen (Autoimmun-)Erkrankungen spielen könnten. Ihre Entdeckung wird als fortlaufende Erweiterung des von Nedergaard gefundenen glymphatischen Systems angesehen.

Aspelund et al. [10] beschrieben den Schlemm-Kanal, der bis dato als venöser Sinus angesehen wurde, als ein lymphähnliches Gefäß. Kipnis [3] legt nahe, dass auch andere venöse Sinus eher lymphatischer Natur sein könnten. Dass man sie so lange nicht entdeckt habe, liege daran, dass sie sehr gut versteckt in den Meningen liegen. Das Kipnis-Labor hat ein Gefäßnetzwerk in der Dura entlang des Sinus sagittalis superior und Sinus transversalis mit direkter Verbindung zu den zervikalen Lymphgefäßen gefunden. Die Drainage erfolgt in den Kanälen entlang der venösen Sinus, Meningealarterien und Hirnnerven sowie der Lamina cribriformis und über eine Absorption des Liquors.

Die lange Zeit als gültig betrachtete Vorstellungsweise des Gehirns ohne Lymphsystem ist somit nicht mehr haltbar.

\section{Liquor cerebrospinalis}

Schon Hippokrates (460-375 v. Chr.) und Galen (130200 n. Chr.) stellten die Hypothese auf, dass es diese Flüssigkeit gibt. Dass die Entdeckung dann erst Swedenborg (1688-1772) zugeschrieben wurde, liegt vermutlich daran, dass zu dieser Zeit der Schädel abgetrennt und ausgeblutet wurde, bevor er seziert wurde. Swedenborg bezeichnete den Liquor als Sitz der Seele, als die spirituelle Lymphe, und wies auch auf eine Verbindung zwischen ihm und dem lymphatischen System hin [11]. Der Liquor wird im jeweiligen Plexus choroideus in den Ventrikeln gebildet, fließt in den Subarachnoidalraum und wird dann über die Granulationes arachnoideae der duralen Sinus bzw. die kranialen Nervenscheiden in die peripheren Lymphgefäße bzw. Venen drainiert ( $\bullet$ Abb. 1). Er entspricht einer Senke für die Aufnahme der in der interstitiellen Flüssigkeit gelösten Stoffe, z. B. Proteine wie Amyloid $\beta$ und endokrine Stoffe. Somit findet durch den Liquor cerebrospinalis die Reinigung des Gehirnparenchyms statt, obwohl er in keinem direkten Kontakt dazu steht.

Im Liquor cerebrospinalis sammeln sich alle Stoffe, die nicht über die Blut-Hirn-Schranke gelangen und ausgeschieden werden können. Lange ging man davon aus, dass dies durch Diffusion geschieht, doch bei genauerer Betrachtung wird deutlich, dass dieser Vorgang viel zu langsam wäre. Laut einer Kalkulation von Cserr [2] würde 


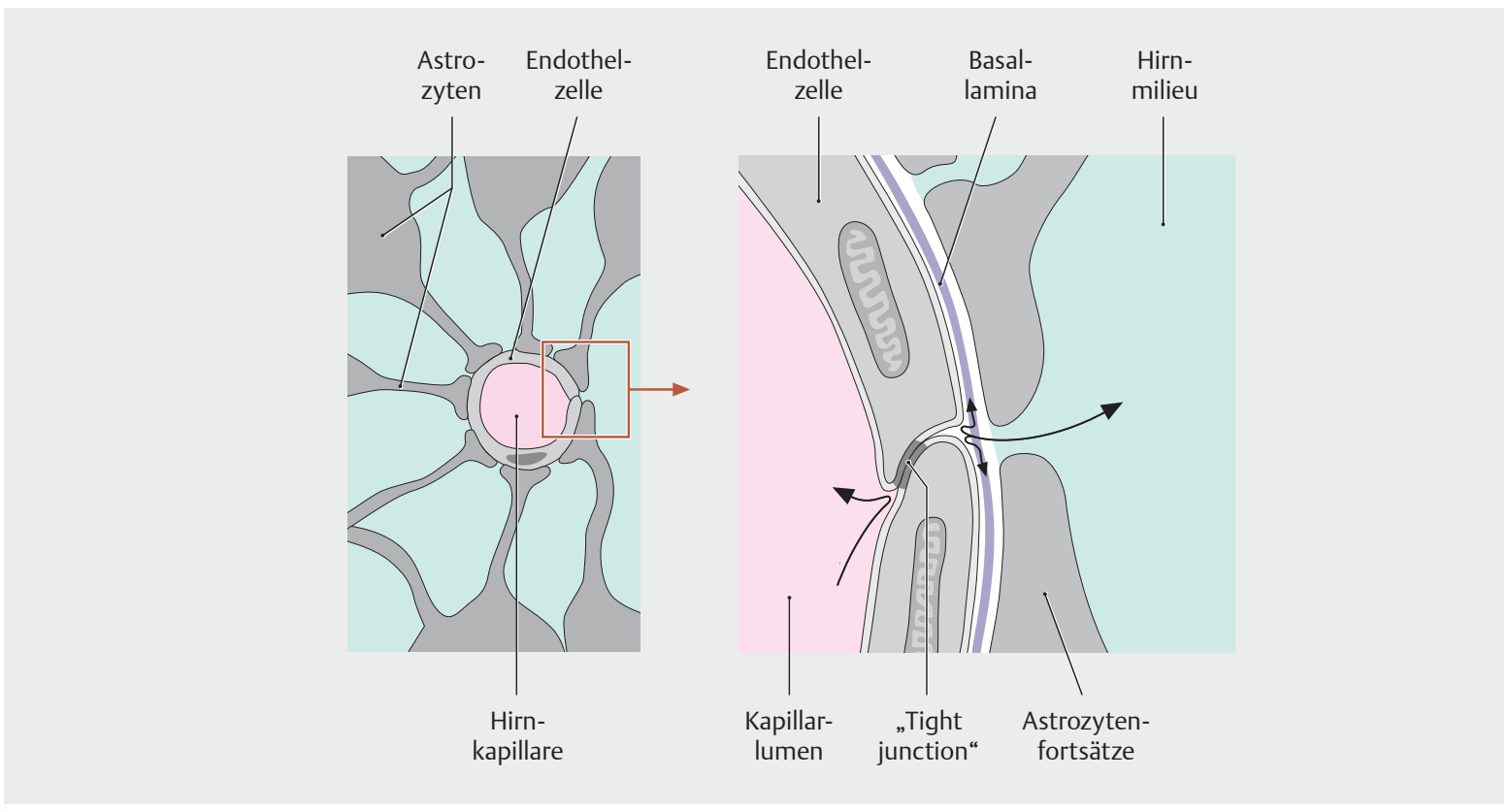

- Abb. 2 Blut-Hirn-Schranke. (Quelle: Schünke M, Schulte E, Schumacher U. Prometheus. LernAtlas der Anatomie. Allgemeine Anatomie und Bewegungssystem. Illustrationen von M. Voll und K. Wesker. 5. Aufl. Stuttgart: Thieme; 2018)

z. B. allein Serumalbumin im Gehirnparenchym 100 Stunden für $1 \mathrm{~cm}$ benötigen. Das heißt, der Abstand zwischen interstitieller Flüssigkeit und Liquor in Ventrikeln und Subarachnoidalraum ist eigentlich zu groß für einen rein durch Diffusion stattfindenden Abtransport makromolekularer Abfallstoffe. Da das Gehirn eine extrem hohe Stoffwechselaktivität hat, muss es andere Mechanismen geben.

Das Gehirn reagiert extrem empfindlich auf homöostatische Schwankungen. Da der Liquor an der Aufrechterhaltung der Homöostase beteiligt ist, würde es an einer entsprechenden Sensitivität für die schnellen homöostatischen Veränderungen mangeln, wenn alles nur auf Diffusion beruhen würde. Cserr et al. [2] schlugen daher vor, dass es sich um einen konvektiven Strömungsschwall der interstitiellen Flüssigkeit vom Gehirnparenchym zum Liquor handeln könnte, wodurch eine effektive Abfallbeseitigung zu gewährleisten wäre. Heute weiß man, dass es einen Bulkflow (= schwallartig) im Liquor gibt, der am schnellsten im Bereich der weißen Materie und perivaskulär ungefähr genauso schnell wie der Lymphfluss in der Peripherie fließt. Es handelt sich daher vermutlich um eine Kombination aus Bulkflow der makroskopischen Anteile durch das Gehirnparenchym zu den Liquorzisternen ( $\triangleright$ Abb. 3) und Diffusion der mikroskopischen Stoffe zwischen perivaskulärem Raum und Interstitium.

\section{Versorgung des Gehirns}

\section{Arterien und Venen}

Die arterielle Versorgung des ZNS erfolgt durch die A. carotis interna sowie die Aa. vertebrales, die sich zur $A$. basilaris vereinigen. Die Gefäße treten durch die Dura und bilden mit der A. cerebri anterior, A. cerebri media und A. cerebri posterior den Circulus arteriosus cerebri (Willisii).

Der venöse Abfluss erfolgt durch Sinus sagittalis superior, Sinus sagittalis inferior, Sinus rectus und Sinus transversus durch das Foramen jugulare (V. jugularis interna) und den Spinalkanal (Batson-Plexus).

\section{Liquor}

Die Arterien des ZNS haben ab ihrem Eintritt durch die Hirnhaut rund um ihre Außenwand einen zusätzlichen, sehr engen Gefäßraum, den perivaskulären Raum (Spatium perivasculare), der für die Blutgefäße im ZNS die Bezeichnung Virchow-Robin-Raum (VRR) trägt. Die innere Wand dieses Hohlraums bilden die Endothelzellen der Blutgefäße, die äußere Wand spezielle plattenförmige Ausläufer von Astrozyten, die Astrozyten-Endfüße, die sich zu einem dichten Geflecht zusammenlagern. Durch den VVR gelangt in einem ständigen Strom - angetrieben durch die vom Pulsschlag ausgelösten Wellenbewegungen der Arterienwände - ein kleiner Teil des Liquors aus dem Zwischenraum zwischen Schädeldecke und Gehirn 


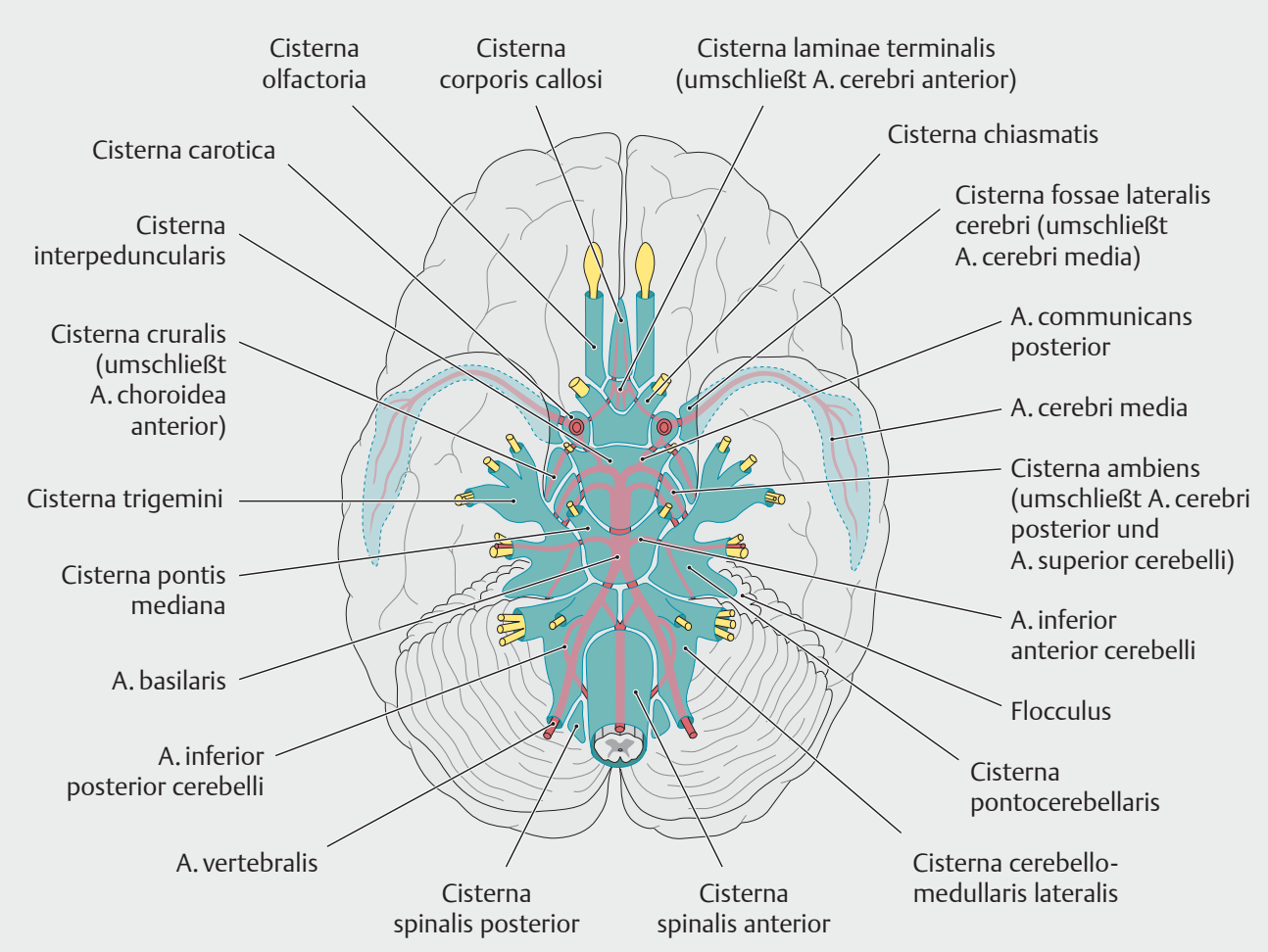

Abb. 3 Liquorzisternen (mit Liquor gefüllte Erweiterungen des Subarachnoidalraums). (Quelle: Schünke M, Schulte E, Schumacher U. Prometheus. LernAtlas der Anatomie. Kopf, Hals und Neuroanatomie. Illustrationen von M. Voll und K. Wesker. 5. Aufl. Stuttgart: Thieme; 2018)

(Subarachnoidalraum oder äußerer Liquorraum) in alle Bereiche des ZNS.

Rund um die leptomeningealen Arterien und Venen befindet sich Pia mater. Im Subarachnoidalraum verlaufen Liquorkanäle zwischen den Blutgefäßen und der Leptomeninxscheide im VRR. Dieser endet mit dem Parenchym des Gehirns.

Eine Vergrößerung des VRR geht oft mit Schlaganfällen, kognitiven Störungen, Demenz, Herzerkrankungen und Schlafstörungen einher [8]. Der VRR kontrolliert den schwallartigen Fluss (Bulkflow) von Interstitialflüssigkeit, was auch mit der Klärung von Abfallstoffen einhergeht.

Der Liquor wandert weiter entlang der Membran um die glatte Muskulatur der arteriellen Gefäße bis hin zur Basallamina der das Gehirn umgebenden Kapillaren. Die durch den Pulsschlag ausgelöste Wellenbewegung der Arterienwände und der hydrostatische Druckgradient stimulieren den sehr schnellen Fluss des Liquors im Subarachnoidalraum des gesamten Gehirns [9]. Er dringt über die perivaskulären Räume entlang der penetrierenden Arterien über die Kapillaren der Basallamina in das Gehirnparenchym ein und tauscht sich entlang dieser perivaskulären Räume mit den umgebenden interstitiellen Fluida aus.
Das heißt, VRR, perivaskulärer Raum und vaskuläre Basalmembran stehen alle untereinander in Verbindung und der Liquor aus den Zisternen fließt dort hindurch. Kleine Stoffe werden schneller zwischen Interstitium und perivaskulärem Raum ausgetauscht (Diffusion), während die großen gelösten Stoffe durch die Astrozyten-Endfüßchen in die Gehirngefäße gelangen. Ein Großteil des Gliagewebes besteht aus Astrozyten. Sie umschließen engmaschig den VRR und bilden somit die Kontaktebene zwischen den neuronalen Synapsen und den die Gehirngefäße komplett umkleidenden Scheiden. Sie spielen eine wichtige Rolle bei Veränderungen im Blutfluss und bei der Abfallbeseitigung. Der durchfließende Liqour wird von ihnen resorbiert und ans Interstitium von Gehirn und Rückenmark weitergeleitet.

\section{Aquaporin}

Eine wichtige und aktive Rolle bei der Klärung der gelösten Stoffe spielen die Wasserkanäle Aquaporin 4 (AQP4) an 50\% der perivaskulären Endfüße der Astrozyten, die an den Kontaktstellen zu den Arterien gelegen sind ( $\triangleright$ Abb. 4). Es handelt sich dabei um transmembrane, also membrangebundene, Kanäle in Gehirn und Ependymwänden der Ventrikel, die eine entscheidende Rolle bei der Regulierung des Wasserflusses in die und aus den Zellen heraus spielen. Sie erhöhen die Diffusions- 


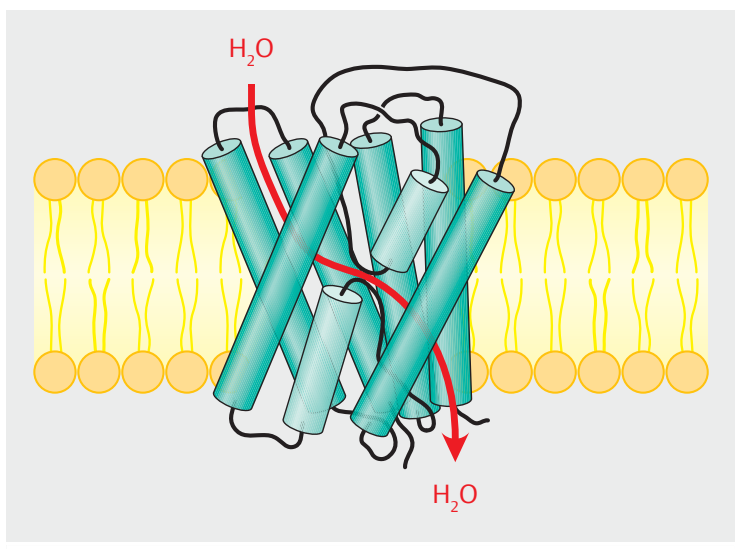

Abb. 4 Aquaporin. (Quelle: Hauser K. Porine. In: Rassow J, Hauser K, Deutzmann R et al., Hrsg. Duale Reihe Biochemie. 4. Auflage. Stuttgart: Thieme; 2016)

permeabilität biologischer Membrane um das 3-10Fache.

Im ZNS findet man 2 Arten von Aquaporinen:

- AQP1 wird von Epithelzellen im Plexus choroideus exprimiert.

- AQP4 wird von Astrozyten exprimiert, insbesondere an den Endfüßchen der zerebralen Gefäße. Es ist für den Austausch von Liquor und Interstitialflüssigkeit essenziell [2].

\section{Lymphsystem des Gehirns}

Die Lymphe erfüllt eine wesentliche Immun- sowie eine Entsorgungsfunktion. Die Lymphgefäße verlaufen parallel zu den Blutgefäßen und transportieren interstitielle Flüssigkeit, Proteine und Stoffwechselprodukte. Da die Proteine für den Erhalt des kolloidosmotischen Drucks und die homöostatische Regulation des Flüssigkeitsvolumens des Körpers zuständig sind, ist ein Abtransport von anfallenden Proteinen sehr wichtig. Fehlt dieser, kommt es zu Ödemen.

Im Bereich der Arteriolen und Kapillaren sickert Flüssigkeit in das Interstitium. Diese ist ein Filtrat des Plasmas. Sie wandert weiter zu den kapillaren Venolen und fließt dann in die Venengeflechte bzw. über den perivaskulären Raum entlang der Venen und verlässt so das durale System und Gehirn. Postkapillär kommt es zum Teil zur Reabsorption in die duralen Sinus bzw. Gefäße, in deren Wänden sich die venenklappenähnlichen Arachnoidalzotten befinden, bzw. drainiert die Lymphe mit den makroskopischen Proteinen letztendlich in die zervikalen Lymphgefäße und von dort zurück in die Venen. So kommt es zur Reinigung über das venöse System und die Abbauprodukte werden über Leber und Nieren entsorgt.
Nicht alle Proteine und Metabolite können die Blut-HirnSchranke passieren. Diese werden über den Liquor entsorgt. Dazu gehören auch Metabolite (Abfallstoffe), Hormone, Lipide und Immunzellen. Auch der Lipidtransport findet über das glymphatische System statt [2]. Kleine lipophile Moleküle, die das gliale Kalziumsignalsystem aktivieren, werden entfernt. Allerdings gehen diese Lymphgefäße nicht ins Gehirnparenchym hinein, sondern liegen an den Außenschichten der Meningen. Daher sind vermutlich die perivaskulären glymphatischen Wege, die Liquorzisternen und die duralen Lymphgefäße alle Teil eines Systems für die Clearance der Metabolite aus dem Interstitium und der Immunüberwachung.

Die meningealen Lymphgefäße verlaufen parallel zu den duralen Sinus und Meningealarterien des ZNS. Sie drainieren in die tiefen zervikalen Lymphknoten. Sie verbinden Immun- und Nervensystem und könnten daher eine Rolle bei Autoimmun- und neurodegenerativen Erkrankungen spielen. Vor der Entdeckung der meningealen Lymphgefäße ging man davon aus, dass das Gehirn den Überschuss an Flüssigkeit, die Proteine und Metaboliten über das glymphatische System, den Liqour über die Lamina cribriformis in die Lymphgefäße der Nasenmukosa und über die Arachnoidalzotten drainiert [10].

Die meningealen Lymphgefäße sind im Vergleich zu den peripheren weniger komplex mit geringerer Verzweigung und kleineren Gefäßen und decken dadurch auch weniger Gewebe ab. Sie sind entlang des Sinus sagittalis dünner und wenig verästelt und entlang des Sinus transversus etwas dicker mit mehr Verästelungen. Lediglich im basalen Anteil des Kraniums finden sich einige wenige Klappen, die den Rückfluss verhindern.

Der Liquor tritt entlang der Arteriolen ins Gehirn ein, die interstitielle Flüssigkeit tritt entlang der Venolen aus und fließt weiter zum Sinus rectus. Diese Erkenntnis beruht auf Versuchen in den 1980er-Jahren mit Katzen und Hunden. Inzwischen wurde dies auch bei Mäusen nachgewiesen. Der Liquor besteht aus glialem Wasser und hat eine lymphatische Funktion (=glymphatisches System). Dies ist im gesamten Gehirn so.

\section{Immunaufgaben des Lymphsystems}

Das Lymphsystem hat mit grundlegenden Aspekten der Organfunktion wie interstitielle Flüssigkeit, Homöostase der Lipide und Proteine sowie Transport von Lymphozyten und antigenpräsentierenden Zellen zu den Lymphknoten zu tun. Das scheint auch im Gehirn entsprechend zu sein. Ebenso wie das periphere Lymphsystem hat das meningeale Lymphsystem eine Immunzellfunktion mit B- und T-Lymphozyten und drainiert das Gewebe. Die Lymphgefäße filtern im Anschluss an das glymphatische System Makromoleküle des Gehirnparenchyms aus. Fehlen die Lymphgefäße, z.B. weil es durch das Fehlen von 
VEGF-C und -D (Vascular Endothelial Growth Factor) in der Embryogenese nicht zur Entwicklung der Lymphgefäße gekommen ist, kann laut Kipnis und Louveau [3] das Gehirnparenchym allerdings eine mangelnde Klärung von Feststoffen kompensieren (Mäuse). Entlang der perivaskulären Wege werden Amyloid $\beta$, Tau-Proteine (werden bei neuraler Aktivität im Extrazellulärraum des Gehirns freigesetzt), Proteine (Albumin) etc. weggeräumt. Ist die Drainage über die sinusoidalen Lymphgefäße geblockt, sammeln sich periphere Immunzellen in der Dura, d. h., sie sind wohl auch an der Überwachung der Immunaktivität im ZNS beteiligt. Periphere Immunzellen kommen viel über den Plexus choroideus in den Liquor und werden durch die mit den Sinus verlaufenden Lymphgefäße abtransportiert.

Es gibt also eine periphere Immunüberwachung des Gehirns, ohne die Privilegien des Immunsystems des ZNS aufzuheben.

\section{Schlaf}

Das Gehirn, dessen Masse nur 2\% des Körpergewichts beträgt, verbraucht ca. $\mathbf{2 5}$ \% der Energie. Das heißt, es fallen auch entsprechend Metabolite an, die abtransportiert werden müssen. Das geschieht durch den Liquor. Iliff et al. [9] haben nachgewiesen, dass der Liquor an der Oberfläche des Gehirns durch das Interstitium entlang der Gefäße durch das Gehirn durchgespült wird und somit Proteine und andere Metabolite ausspült.

Das glymphatische System entsorgt gemeinsam mit dem lymphatischen System die Metabolite des ZNS. Die Hauptaktivitätszeit ist nachts. 90-95\% der Entsorgung finden während der Tiefschlafphasen statt. Außerdem ist der Fluss nachts schneller, vermutlich auch dadurch bedingt, dass die Zellen nachts schrumpfen und somit bis zu 60-65\% mehr Platz zum Durchspülen (Bulkflow) gegeben ist $[2,4]$. Der Fluss dringt auch tiefer ins Gehirn ein, statt nur an der Oberfläche wie im Wachzustand zu fließen [4]. Laut Xie et al. [4] findet der beste Abtransport in Seitenlage statt.

Im Wachzustand sind die Zellen auch durch das Hormon Noradrenalin mehr geschwollen. Dieses erhöht den kortikalen Tonus und die Aufmerksamkeit (d.h. auch: weniger Noradrenalin $=$ mehr Durchfluss $=$ mehr Reinigung) . Blockt man kortikale Noradrenalinsignale im Wachzustand, wird das extrazelluläre Volumen größer und dadurch die glymphatische Aktivität erhöht.

Fasst man dies alles zusammen, bedeutet dies:

- Da die Hauptaktivität des glymphatischen Systems, nämlich 95\%, im Schlaf stattfindet, könnte mangelnder Schlaf zu einer verminderten Reinigung des Gehirns führen, da Metabolite nicht ausreichend abtransportiert werden.
- Die Klärung des glymphatischen Systems findet während der Tiefschlafphasen statt.

- Das Interstitium (= EZR) vergrößert während des Schlafs seinen Raum, da die Zellkörper schrumpfen. Das Gesamtvolumen des Interstitiums, das im Wachzustand $14 \%$ beträgt, erhöht sich auf $24 \%$, d. h. es stehen über $60 \%$ mehr Raum für den Transport der Fluida zur Verfügung.

- Ein möglicher Regler für das Volumen des Interstitialraums ist Noradrenalin, das einer der Hauptmodulatoren des Wachzustands ist. Wäre dem so, dann wäre es auch mit an der Effektivität des glymphatischen Systems beteiligt.

- Die Reinigung erfolgt durch Bulkflow (schwallartig) und Diffusion, je nach chemischer Zusammensetzung und Umgebung. Die meisten gelösten Substanzen werden über Diffusion ausgefiltert.

Führt man diesen Gedanken weiter, so sind Schlafstörungen möglicherweise nicht unbeteiligt am Auftreten neurodegenerativer Erkrankungen und pathogener Formen der Tau-Proteine. Diese kommen v.a. in Neuronen des ZNS vor und stabilisieren die Mikrotubuli. Eine geringe Anzahl findet sich auch in den Astro- und Oligodendrozyten. Defekte Tau-Proteine führen zu einer Destabilisierung der Mikrotubuli, z.B. bei Morbus Alzheimer und Morbus Parkinson [7].

Störungen des glymphatischen Systems finden sich z. B. bei Druckverlust im Schädel, Schlaganfall, Subarachnoidal- oder intrakranialen Blutungen. Bedingt z. B. durch Traumata (Schädel-Hirn-Trauma, Ischämie), Morbus Alzheimer (Ansammlung von Proteinen) oder entzündliche Vorgänge (z. B. multiple Sklerose) können sich Ödeme entwickeln. Kommt es zu einem Druckverlust in der Schädelhöhle, wird die glymphatische Zirkulation gestört und es kommt zu einer nichtselektiven Lipiddiffusion, einer Ansammlung der intrakranialen Lipide und einer pathologischen Signalgebung für die Astrozyten. Man könnte die Funktion mit der der peripheren intestinalen Lymphgefäße vergleichen, welche die Lipide zur Leber abtransportieren.

Die Annahme, dass nicht nur Vorgänge im Zellinneren, sondern auch im Interstitium an degenerativen neurologischen Erkrankungen wie z. B. Morbus Alzheimer beteiligt sind, verdeutlicht, wie wichtig eine gute Reinigung mithilfe des glymphatischen Systems wahrscheinlich ist [7]. In diesem Falle geht es um den Abtransport fehlgefalteter Proteine und um Amyloid $\beta$ (sog. senile Plaques). Die Produktion dieses Peptids findet normalerweise in jungen Gehirnen statt und wird da auch abtransportiert. Bei Morbus Alzheimer hingegen lagert es sich aufgrund des mangelnden Abtransports in den Zwischenzellräumen ab und bildet Plaques, die zu einem Verlust an neuronalem Gewebe und zur Gehirnatrophie führen. Bei der amyotrophen Lateralsklerose findet man eine An- 
häufung des fehlgefalteten Enzyms SOD1. Es wurde die Hypothese aufgestellt, dass eine Fehlfunktion des glymphatischen System die Ursache für diese Erkrankung sein könnte [7].

\section{Altern bzw. Neurodegeneration}

Während des physiologischen Alterungsprozesses wird der Zustrom von frischer Flüssigkeit durch die schwächer werdende Bewegung der Arterien beeinträchtigt. Es können nicht mehr genug Abfallstoffe geklärt werden. Es kommt zu einer Akkumulation von fibrillären Proteinaggregaten, z.B. extrazellulären senilen Plaques einschließlich Amyloid $\beta$ und intrazellulären neurofibrillären Bündel einschließlich hyperphosphorilierten Tau-Proteinen bei Morbus Alzheimer. Im alternden Nagetiergehirn zeigte sich, dass eine beeinträchtigte glymphatische Klärung und ein Verlust von perivaskulären AQP4 zu einer vermehrten Proteinansammlung und dadurch zu einer Neurodegeneration führten [2].

Das Gleiche findet man auch bei anderen neurodegenerativen Erkrankungen wie Morbus Parkinson, bei denen Ablagerungen auftreten oder entzündliche Prozesse vermutet werden. Möglicherweise sind Schlafstörungen im Alter mit ein wichtiger Grund für diese mangelnde Reinigung.

Gehirnerschütterungen erhöhen das Risiko für Demenz und Alzheimer. Alle Kontaktsportarten können z. B. zu einer traumatischen Enzephalopathie führen. Man findet hier perivaskuläre Ablagerungen von Tau-Aggregaten in der oberflächlichen Kortexschicht. Bei Nagern sind die glymphatischen Wege und AQP4-Lokalisationen in diesen Fällen chronisch geschädigt, wodurch es zu einer verminderten Klärung kommt $[1,6]$.

Da die physiologischen Mechanismen des zerebralen glymphatischen und lymphatischen Systems noch weitgehend unerforscht sind, kann momentan noch nicht gesagt werden, wie groß der Einfluss einer Dysfunktion auf neurovaskuläre, neurodegenerative und neuroinflammatorische Prozesse tatsächlich ist.

\section{Osteopathische Überlegungen}

Berücksichtigt man diese neuen Erkenntnisse hinsichtlich der Versorgung und Reinigung des Gehirns, so gewinnen Herangehensweisen über das fluidische System eine weitere wichtige Perspektive. Die Anregung der intrakranialen venösen Drainage und Zirkulation einschließlich der Beurteilung des Schlemm-Kanals bei Augenproblemen, Techniken wie der CV4 und die Berücksichtigung des Einflusses des autonomen Nervensystems sind beispielsweise wunderbare Möglichkeiten, auf das glymphatische System einzuwirken. Das autonome Nervensystem gewinnt besondere Bedeutung, wenn man z. B. an Schock- geschehen, Trauma und chronische Stresssymptome beim posttraumatischen Belastungssyndrom denkt.

\section{Autorinnen/Autoren}

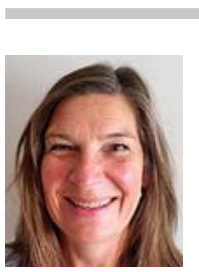

\section{Susanne Dick-Wallace}

schloss 1987 ihre Ausbildung als Physiotherapeutin ab, ist seit 2002 Osteopathin und seit 2003 Heilpraktikerin. Seit 2007 lebt und arbeitet sie in eigener Praxis in Kanada, ist aber auch weiterhin sowohl als Osteopathin als auch als Übersetzerin osteopathischer Fortbildungen regelmäßig in Deutschland tätig. Sie ist Table Trainerin bei Dr. James Jealous und gibt selbst Workshops in Deutschland und Kanada.

Korrespondenzadresse

Susanne F. Dick-Wallace D.O.

Box 717

Sangudo, AB TOE 2A0

Canada

suefdick@web.de

Literatur

[1] Caplan LR et al. Primer on cerebrovascular diseases. 2. Aufl. Elsevier: Academic Press; 2017

[2] Plog BA, Nedergaard M. The glymphatic system in CNS health and disease: past, present and future. Annu Rev Pathol 2018; 13: 379-394

[3] Louveau A et al. Structural and functional features of central nervous system lymphatic vessels. Nature 2015; 523: 337341

[4] Xie L et al. Sleep drives metabolite clearance from the adult brain. Science 2013; 342 (6156): 373-377

[5] Shaw G. New Study Suggests Brain Is Connected to the Lymphatic System. What the Discovery Could Mean for Neurology. Neurology Today 2015; 15 (13): 1,9-10

[6] Hitscherich K et al. The Glymphatic-Lymphatic Continuum: Opportunities for Osteopathic Manipulative Medicine. JAOA 2016; 3: 170-177

[7] Konnikova M. Goodnight. Sleep Clean. NYT, 11.1.2014

[8] alzforum.org/news/research-news/sleep-and-brain-cleansingfresh-insights-regulation-and-disruption

[9] Iliff J] et al. Brain-wide pathway for waste clearance captured by contrast-enhanced MRI. J Clin Invest 2013; 123 (3): 12991309

[10] Aspelund A. A dural lymphatic vascular system that drains brain interstitial fluid and macromolecules. J Exp Med 2015; 212 (7): 991-999

[11] Fuller DB. Osteopathy and Swedenborg. Bryn Athyn: Swedenborg Scientific; 2012

[12] Brock E] et al. Swedenborg and his Influence. Bryn Athyn: Academy of the New Church; 1988

\section{Bibliografie}

DOI https://doi.org/10.1055/a-1084-8564 DO - Deutsche Zeitschrift für Osteopathie 2020; 18: 32-38 (c) Georg Thieme Verlag KG Stuttgart · New York ISSN 1610-5044 